\title{
Overconsumption, credit rationing and bailout monetary policy: A Minskyan perspective
}

\author{
Matthieu Charpe*, Peter Flaschel**, \\ Christian Proaño***, Willi Semmler ${ }^{* * * * * * * *}$
}

\begin{abstract}
We consider a Keynes-Goodwin model of effective demand and the distributive cycle where workers purchase goods and houses with a marginal propensity significantly larger than one. They therefore need credit, supplied from asset holders, and have to pay interest on their outstanding debt. In this initial situation, the steady state is attracting, while a marginal propensity closer to one makes it repelling. The stable excessive overconsumption case can easily turn from a stable boom to explosiveness and from there through induced processes of credit rationing into a devastating bust. In such a situation the central bank may prevent the worst by acting as creditor of last resort, purchasing loans where otherwise debt default (and bankruptcy regarding house ownership) would occur. This bail-out policy can stabilize the economy and also reduces the loss of homes of worker families.
\end{abstract}

JEL classification: $E_{24}, E_{31}, E_{32}$

Keywords: mortgage loans, booms, debt default, busts, creditor of last resort

* International Labor Organization, Genéve, Switzerland

** Bielefeld University, Bielefeld, Germany

*** Macroeconomic Policy Institute, Düsseldorf, Germany

**** New School University, New York, USA

***** We would like to thank two anonymous referees for valuable comments and suggestions on a previous draft of this paper. All remaining errors are, of course, of our responsibility.

Correspondence Address:

Dr. Christian Proaño, Macroeconomic Policy Institute (IMK) at the Hans Boeckler Foundation, HansBoeckler-Strasse 39, 40476 Duesseldorf, Germany, e-mail: christian.proano@web.de.

Received I5 January 2009, accepted 04 May 2009

(C) INTERVENTION 6 (2), 2009, 247-270 


\section{Introduction}

"Success breeds disregard of the possibility of failures. The absence of serious financial difficulties over a substantial period leads [...] to a euphoric economy in which short-term financing of long term positions becomes the normal way of life. As previous financial crisis recedes in time, it is quite natural for central bankers, government officials, bankers, businessmen and even economists to believe that a new era has arrived «. (Minsky 1982: 213).

As it is widely acknowledged, the current financial crisis in the USA was originally triggered by an excessive expansion of mortgage loans to households in order to purchase real estate. The main difference with the traditional Minskyan crisis and the actual US financial crisis is here that borrowers are not firms but households. Indeed, while for example the Japanese crisis was characterized by firms and banks borrowing to invest in real estate, and the East Asia crisis was characterized by firms and banks borrowing foreign denominated debt, in the current crisis, financial fragility leading borrowers from hedge- to speculative- and Ponzi positions have involved the households' income and interest payments significantly.

There are three main factors which explain the large increase in mortgage credits over the last years in the US economy: Competition between financial institutions, the interaction between real estate price and credit awarding as well as the proliferation of securitization. Jointly, these three elements induced banks to relax screening and monitoring of borrowers and to increase the quantity of credit supplied to households. First, in the aftermath of the crash of the dotcom bubble in 2000 and in an environment characterized by low interest rates, banks, under the pressure of financial intermediaries such as hedge funds, found in mortgage debt a highly profitable business. Second, increasing real estate prices (and the expectation of further increases) contributed significantly to an excessive expansion of credit to, under otherwise normal circumstances, borrowers with insufficient creditworthiness. The increasing collateral's value reduced default risks and led banks to increase credit, along the lines of the financial accelerator model. And lastly, the widespread increase of securitization up to an unprecedented level lowered dramatically the practice of borrowers' screening and monitoring by banks (in 2006, securitization concerns $87 \%$ of prime mortgages and $75 \%$ subprime mortgages (Ashcraft/Schuermann 2007). ${ }^{\mathrm{I}}$

The widespread increase of securitization had dramatic consequences for the credit markets: On the one hand, the belief that credit risk could be transferred without further problems to the market led banks to expand credit supply, leading to a worsening of the average quality of loans. In addition, securitization reduced the need of banks to increase their own funds following credit expansion. In a traditional banking sector, banks are exposed

I Securitization aims at transforming loans into liquid assets in order to transfer the credit risk to the market. Mortgages with different qualities are mixed together and the resulting financial products are sold to the market through SPVs (special purpose vehicules) in the form of CDOs (collateralized debt obligations) or RMBSs (residential mortgage backed securities) for instance. 
to credit risk and must provision reserves to cover the risk accordingly. As risks were transferred to the market, banks were not forced to provision for risks. Although securitization was supposed to reduce banks exposure to risk, securitization induced banks to take more risks, in fact. As a result, low income households were able to access credits, the so-called subprime mortgages. While subprime borrowers accounted for nine per cent of borrowers in 2000, this proportion increased to 20 per cent in 2006 (Dell'Ariccia et al. 2008). This large increase in subprime mortgage debt produced a deterioration of the income of these type of households, not yet considered in the present model, and soaring debt default.

In this paper, however, we leave aside the role played by real estate prices, risky financial innovations and subprime debt default. We study instead as a baseline exercise the macrodynamic features of worker household debt accumulation and debt crisis, our main focus being the understanding of the dynamics between worker households' overconsumption and debt, their interest payments, their real wage dynamics and effective demand.

For this we set up a basic model characterized initially by stable, but later on by unstable excessive overconsumption of worker households. Credit then becomes rationed and the economy diverges - if this occurs - even more from its initially stable steady state position. We then consider actions that rescue the economy from this instability scenario and lead it back to economic stability, based on investment stimuli and monetary policy regarding the loan and the default rate on the credit market.

Our focus on overconsumption and debt is however not new altogether: Palley (I994) and Dutt (2003 and 2006) are earlier studies in the Keynesian macrodynamics tradition which have analyzed the short- and long-run effects of workers' overconsumption and debt. Palley (1994) focuses on the double-sided character of workers' indebtedness, showing that an increase in workers' indebtedness increases aggregate demand, on the one hand, due to higher consumption, but is also likely to depress it, on the other hand, due to the larger debt-service payments from debtors to creditors (which as traditionally assumed in neoKaleckian models, have a lower propensity to consume). Dutt (2003 and 2006) focuses on the short- and long-run effects of such a development. ${ }^{2}$ However, the (un-)sustainability of sconspicuous consumption was not explicitly addressed in both approaches. In contrast, the innovation of this paper is the explicit analysis of credit rationing to workers as a result of over-indebtedness as well as the role of (bailout) monetary policy for economic stabilization after such a credit crisis.

The starting framework of the paper is a Goodwin model of the distributive cycle with a single commodity (that can be used for consumption and investment purposes, including housing investment), in which households' debt is introduced in order to assess when it adds stability and when instability to the baseline dynamics. Moreover, the economy is restricted by a Keynesian effective demand function, since the act of saving (by asset holders) differs from the act of investment (by firms). We thus here integrate a Goodwin type

2 Dutt shows that the effect of higher consumer indebtedness on aggregate demand is always positive in the short run but can become ambiguous in the long run. 
conflict about income distribution with a Keynesian goods demand framework, which is replacing the original supply side dynamics of the Classical growth cycle.

As it will be stressed on several occasions, the central features of this macrodynamic model, and its application to the study of credit markets and boom-bust cycles, are the mechanisms generating overheated or depressed goods markets positions. Furthermore, in contrast to the tradition which stresses the clearing of all markets at each instance of time, ${ }^{3}$ in our modeling approach disequilibrium situations are the main driving forces of an integrated wage/price dynamics. Moreover, disequilibrium effects in financial markets are often generated by over-leveraging in the real sector, here in the household sector as well as in the financial sector of the economy.

Due to the fact that in our modeling approach the stability of the analyzed dynamical system is not imposed ad initio by rational expectations assumptions (which requires that the economy always sjumps to some stable manifold and therefore always converges to the steady state after any type of shock), its stability properties are based on the relative strength of the interacting macroeconomic and financial feedback channels, through a variety of different macroeconomic repercussions, showing that there are indeed different (and also valid) possibilities to specify and analyze the dynamics of the macro economy in a rather different way than in the standard DSGE framework.

In view of the above quotation from Minsky's work, see also Minsky (I992), it may therefore well be - if the last occurrence of an eventually destabilizing overconsumption scenario is sufficiently back in time (and also covered up by important historical events such as the breakdown of Eastern type socialism) - that the initially stable excessive overconsumption reappears and that the whole process starts again, though possibly in a different historical garment. The above quotation from Minsky's work is therefore characteristic for the four stages of macrodynamic evolution we are considering in this paper, though the macro-model we are using is still a very basic one, with commercial banking just represented through asset holding households, with central banking only considered in very partial and stylized form, and with specific historical events, such as particularly vulnerable subprime lending still absent.

\section{A Keynes-Goodwin model with mortgage loans and debt default}

The model of this section consists of two household types: workers and capitalists, who are also the owners of firms.

Workers have a real wage income $Y_{w}$ which they totally spend on goods consumption $c_{g} Y_{w}$ and the purchase of houses $c_{h} Y_{w}$, part of which is financed through loans from the capitalists. Their combined marginal propensity to consume: $c=c_{g}+c_{h}$ is therefore assumed to be larger than one. The workers' real wage income is however reduced through the interest they have to pay on their actual real loans $\Lambda_{w}^{a}$ and the excess of their spending over this 
income determines the amount of new loans $\dot{\Lambda}_{w}$ they need for their intended purchase of new houses. Since the model allows for debt default this rate of change is however not the rate of change of actual loans.

We assume a closed economy and do not consider a fiscal authority in this paper. Moreover, we treat commercial banking by way of asset holders' behavior only. Central banking is considered in a final section and in a very partial way solely. In equations the model reads as follows:

$$
\begin{aligned}
& \text { Workers: } \\
& Y_{w}=v Y-i \Lambda_{w}^{a}, \quad v=\frac{\omega L^{d}}{Y}=\frac{\omega}{z}, \\
& C_{w}=\left(c_{g}+c_{h}\right) Y_{w}, \quad c=c_{g}+c_{h}>1, \\
& \dot{\Lambda}_{w}=(c-1) Y_{w}=-S_{w}, \\
& \dot{K}_{w}=c_{h} Y_{w}-\varphi_{b} K_{w}, \quad \varphi_{b} K_{w}=\varphi_{d} \Lambda_{w}^{a}, \\
& \hat{L}=n=\text { const. }
\end{aligned}
$$

We denote in these equations the real wage by $\omega, z$ is labor productivity and $v$ is the wage share. Labor supply $L$ is subject to natural growth (with a constant rate $n$ ) and employment $L^{d}$ is determined in the sector of firms (see below). The symbol $i$ denotes the loan rate on worker's real debt $\Lambda_{w}^{a}$. The demand of workers of new loans is here not subject to credit rationing (by capitalists), though there is debt default of amount $\varphi_{d} \Lambda_{w}^{a}$ that depends on the loan rate and that is associated in this paper with a depreciation of the stock of houses of the amount $\varphi_{d} K_{w}$ (this assumption intends to represent to a certain degree the triggering mechanism of the current mortgage crisis in the US economy). This loss of housing capital reduces of course the rate of change of the housing stock of workers as shown in Equation $4 .{ }^{4}$ Note finally that the consumption function of workers is the only behavioral assumption that is made in this module of the model.

$$
\begin{aligned}
& \text { Asset Holders: } \\
& \begin{array}{l}
Y_{c}=r K+i \Lambda_{w}^{a}, \quad r=\frac{Y-v Y}{K}-\delta, \\
C_{c}=0 \quad\left[S_{c}=Y_{c}\right], \\
\dot{\Lambda}_{w}^{a}=\dot{\Lambda}_{w}-\varphi_{d} \Lambda_{w}^{a}, \\
W=R+K+\Lambda_{w}^{a}, \\
\dot{R}=Y_{c}-\dot{\Lambda}_{w}-I=S_{c}+S_{w}-I=S-I .
\end{array}
\end{aligned}
$$

4 This, however, is not a central assumption of the model, but helps to avoid the consideration of a resale market for such housing stocks. 
Capitalists - as substitutes for the commercial banking system - do not consume but use instead their savings $S_{c}$ (consisting of their real profit income $r K$, with $r$ the rate of profit of firms $-\delta$ being the depreciation rate of the capital stock -, and their interest income) to a) award new loans $\dot{\Lambda}_{w}$ to workers, b) to award new capital goods to firms, or c) to build up their reserve holdings $\dot{R}$. They do not ration workers and firms with respect to their financing decisions, being thus completely accommodating with respect to their reserves accumulation in the formulation of the model of this section. They are therefore passive funds suppliers, who just get the loan rate on their credit supply (without intermediation by commercial banks), and are thus fairly simple substitutes for such >commercial banks They, however, set the loan rate for the credit market which by and large is an exogenous variable in this paper (see however Section 6 for an exception). We stress, however, that even in such a basic scenario there is room for the occurrence of booms and busts, which may be much more complicated to analyze if asset holding institutions behave in a more active way. Note finally that the paper abstracts from a resale market for houses and thus cannot say anything on the occurrence of booms and busts in such a market (as they preceded the subprime crisis in the US economy).

The sector of firms is also still formulated in a very simple manner. Firms produce output according to effective demand $Y$ (by means of a fixed proportions technology with $y^{p}$ the potential output-capital ratio and $L^{d}=Y / z$ their employment function - with a given labor productivity $z$ ). Their rate of net investment $I / K^{6}$ is determined by the excess of their rate of profit $r$ over the steady state rate of interest $r_{o}$. Moreover, the trend term in their investment behavior is simply given by the natural rate of growth in order to avoid the discussion of how natural growth and capital stock growth adjust to each other. ${ }^{7}$

Firms:

$$
\begin{aligned}
& u=Y / Y^{p}, \\
& y^{p}=Y^{p} / K=\text { const. } \\
& z=Y / L^{d}=\text { const. } \\
& I / K=\beta_{r}\left(r-r_{o}\right)+n,
\end{aligned}
$$

with $u$ representing the capacity utilization rate.

The present paper concentrates on the interaction between indebted workers and credit supplying asset holders (as a substitute for commercial banks). The sector of firms is there-

5 We will assume goods market equilibrium below $S=S_{c}+S_{w}=I$ in terms of net savings and investment and can show then that the change in $R$ is always zero. We therefore ignore the variable $R$ altogether by setting it equal to zero in the following.

6 Depreciation $\delta K$ is retained by firms as means of replacement for worn out capital goods.

7 We will ignore in this paper all effects that can result from changes in the rate of capacity utilization of firms. This would demand the integration of a wage price spiral as considered in Flaschel/ Krolzig (2006) from the theoretical as well as from an empirical point of view. Such a wage price spiral is also needed when the steady state assumption $y_{o}=y^{p}$, see below, is to be derived as an implied condition. 
fore considered as fairly tranquil and not subject to volatile investment behavior. There is in particular no debt financing of investment. Instead it is assumed throughout the paper that asset holders directly invest part of their income into real capital stock formation and this at a rate that is smaller than one as the impact of excess profitability (or loss): $r-r_{o}$ is concerned. This gives rise to real growth dynamics of a Goodwin (1967) type profit squeeze mechanism that can be either wage-led or profit-led, depending on the Keynesian effective demand curve (or IS-curve) the model exhibits. The debt feedback chain of the economy is therefore integrated with such a Keynes-Goodwin growth cycle model in this paper and investigated with respect to the consequences it has in such a framework. We have to admit here that we do not yet integrate state of confidence considerations as in Taylor/O'Connell (1989), as far as investment behavior is concerned, and there is also not yet an explicit impact of the debt to capital ratio onto the behavioral equations of the model, apart from its appearance in the budget equations of households. Such issues must be left for future research.

The final law of motion of this model of fluctuating growth and indebtedness in the housing sector concerns the real wage dynamic that drives the real part of the economy in the form of a real wage Phillips curve (a conventional textbook Phillips curve with myopic perfect foresight regarding price inflation):

Real Wage Adjustment:

$$
\hat{\omega}=\beta_{w e}(e-\bar{e}) \text {. }
$$

As before we denote by $\hat{x}$ the growth rate of a variable $x$, i.e., here of real wages. The variable $e=\frac{L^{d}}{L}$ denotes the rate of employment, with $Y$ the demand driven output level of firms. Real wages are driven by demand pressure $e-\bar{e}$ on the labor market (with $\bar{e}$ the normal rate of employment).

The $3 \mathrm{D}$ dynamics implied by the model of the preceding section to be investigated in this section in a special case, is based on a Phillips curve distributive cycle mechanism interacting with a Goodwin (1967) type growth dynamics and a law of motion for the debt to capital ratio of workers:

$$
\begin{aligned}
& \hat{v}=\beta_{w e}(y / l-\bar{e}), \quad y=\frac{Y}{K}, l=\frac{z L}{K}, v=\frac{\omega}{z}, \\
& \hat{l}=-\beta_{r}\left(r-r_{o}\right), \quad r=\frac{Y-v Y}{K}-\delta=y(1-v)-\delta, l=z L / K, \\
& \dot{\lambda}_{w}^{a}=(c-1)\left(v y-i \lambda_{w}^{a}\right)-\left(\beta_{r}\left(r-r_{o}\right)+n+\varphi_{d}\right) \lambda_{w}^{a}, \quad \lambda_{w}^{a}=\frac{\Lambda_{w}^{a}}{p K},
\end{aligned}
$$

with the following goods market equilibrium or IS expression for the output capital ratio:

$$
y=\frac{c i \lambda_{w}^{a}+\beta_{r}\left(\delta+r_{o}\right)-n-\delta}{\left(c-\beta_{r}\right) v+\beta_{r}-1}=\frac{N(\text { umerator })}{D(\text { enominator })}, \quad y=\frac{Y}{K} .
$$

This IS curve is easily obtained from the goods market equilibrium equation 


$$
Y=c\left(v Y-i \Lambda_{w}^{a}\right)+\left(\beta_{r}\left(r-r_{o}\right)+n+\delta\right) K
$$

by transforming it into intensive form and by solving it for the output capital ratio $y$. Note again that the budget equations of the two types of households of the model imply $\dot{R}=0$ if IS-equilibrium is assumed. ${ }^{8}$ The law of motion for the labor capital ratio $l$ is easily obtained by means of the standard rules for growth rate calculations. And the law of motion for the wage share $v$ is a simple consequence of our real wage Phillips curve when labor productivity is assumed as constant.

We have still a further law of motion, for the ratio $k_{w}=K_{w} / K$ (which is however also not feeding back into the above dynamics), which reads:

$$
\hat{k}_{w}=c_{h}\left(v y-i \lambda_{w}^{a}\right) / k_{w}-\left[\beta_{r}\left(r-r_{o}\right)+n\right]-\varphi_{d} \lambda_{w}^{a} / k_{w} .
$$

This equation shows how bankruptcy of worker households accompanies their debt default concerning credit they have obtained from the capitalists. Of course these two laws of motion should feedback into our baseline dynamics in future extensions of the model. Note here also that there is no resale market for houses in the present formulation of the model so that houses can be treated as consumption goods and need not be classified as investment goods, as it is done in the system of national accounts. By assumption part of the housing stock has been financed by mortgages, and may therefore be subject to bankruptcy if there is debt default occurring with respect to such loans.

\section{I The reference balanced growth path}

Under the assumption that the risk premium is given in an appropriate way (to be determined below), the (in general locally uniquely determined) interior steady state of the dynamics ( $16-18)$ is given by: ${ }^{?}$

$$
\begin{aligned}
& y_{o}=y^{p}, \quad e_{o}=\bar{e}: l_{o}=y_{o} / \bar{e}, \quad y_{w o}=\frac{y_{o}-\delta-n}{c} \quad \lambda_{w o}^{a}=\frac{c-1}{n+\varphi_{d}} y_{w o}, \\
& v_{o}=\frac{y_{w o}+i \lambda_{w o}^{a}}{y_{o}}=\frac{y_{w o}}{y_{o}}\left(1+i \frac{c-1}{n+\varphi_{d}}\right), \quad r_{o}=\left(1-v_{o}\right) y_{o}-\delta .
\end{aligned}
$$

The first two steady state values are set exogenously. The goods market equilibrium then gives the equation for workers' income, which in turn can be used to determine the steady state debt ratio (by setting $\dot{\lambda}_{w}^{a}=0$ ). The steady wage share is then obtained form the defi-

8 On this basis we have

$\dot{R}=r K+i \Lambda_{w}^{a}-\dot{\Lambda}_{w}-I=r K+i \Lambda_{w}^{a}-\left(C-\left(v Y-i \Lambda_{w}^{a}\right)\right)-I=Y-\delta K-C-I=0$.

9 The condition $y_{o}=y^{p}$ is an assumption that can only be proved to be valid if a more complete framework is used that allows to integrate inflation and anti-inflationary monetary policy. The condition on $r_{o}$ is therefore here used as a substitute for the introduction of a larger theoretical framework. 
nition of workers' income. Finally, the target rate of profit $r_{o}$ is adjusted in such a way that this wage share allows for $i=0$.

If one assumes as special case $i=\mathrm{n}+\varphi_{\mathrm{d}}$ one gets in particular:

$$
\begin{aligned}
& y_{o}=y^{p} \quad e_{o}=\bar{e}: l_{o}=y_{o} / \bar{e}, \quad y_{w o}=\frac{y_{o}-\delta-n}{c}, \quad i \lambda_{w o}^{a}=(c-1) y_{w o}, \\
& v_{o}=\frac{y_{w o}+i \lambda_{w o}^{a}}{y_{o}}=\frac{c y_{w o}}{y_{o}}=\frac{y_{o}-\delta-n}{y_{o}}>0, \quad r_{o}=\left(1-v_{o}\right) y_{o}-\delta=n .
\end{aligned}
$$

which ensures that $v_{o} \in(0,1)$ holds true and $r_{o}=n$, the so-called Cambridge equation (for the case $\left.s_{c}=1\right)$. Note that this situation implies that the steady state rate of profit $r_{o}$ is then equal to the rate of return on loans $\frac{i \lambda_{w}^{a}-\varphi_{d} \lambda_{w}^{a}}{\lambda_{w}^{a}}$. The analysis that follows will investigate, with the exception of cases where the loan rate is assumed to be close to zero (see also the section on monetary policy) cases that are close to this special situation. ${ }^{\text {IO }}$

We have already indicated that (some) parameters have to be chosen such that meaningful steady state solutions are obtained. Moreover we restrict the consideration of the dynamics to cases where the rate of profit stays positive and thus do not add further nonlinearities to the model that will guarantee this endogenously (see Flaschel 2009: ch. 4 for the addition of such behavioral nonlinearities). We however allow for cases where $c-1<0$ holds, where therefore asset holders borrow from workers to finance the investment of their firms (close to the steady state). The debtor creditor relationship between asset holders and workers may therefore change, but the former will not go bankrupt as long as their income $Y_{c}$ stays positive.

\section{Excessive overconsumption: \\ From an attracting steady state situation to instability}

\section{I The stable excessive overconsumption case}

We consider in the following the case of a strongly wage-led goods demand

$$
Y^{d}=C+I=c\left(v Y-i \Lambda_{w}^{a}\right)+\beta_{r}\left([(1-v) Y-\delta K]-r_{o} K\right)+(n+\delta) K,
$$

i.e. specifically the case

$$
c>1+\left(1-\beta_{r}\right)\left(1-v_{o}\right) / v_{o}>1>\beta_{r} \quad \text { if } \quad c>1>\beta_{r}>\frac{1-c v_{o}}{1-v_{o}},
$$

IO It is obvious from the above that the steady state values are of meaningful size. They are also meaningful in the border case $i=0$ (keeping $n+\varphi$ fixed). Note moreover again, that the value of $r_{o}$ has been adjusted in such a way that the assumption $\beta_{r}()=0$ is fulfilled at the obtained steady state values. 
where obviously $Y^{d}$ depends positively on the wage share $v$. In this case the Goodwin subcycle is characterized by stability and the marginal propensity of workers to consume is very excessive, and maybe empirically beyond reasonable sizes. We therefore consider this starting case an extreme situation of excessive overconsumption backed up by unrestricted loans. We have in this case that the denominator $D$ in the IS curve is positive and thus also have a positive numerator $N$ when evaluated at the steady state value of $\lambda_{w}^{a}$, namely

$$
y=\frac{c i \lambda_{w}^{a}+\beta_{r}\left(\delta+r_{o}\right)-n-\delta}{\left(c-\beta_{r}\right) v+\beta_{r}-1}=\frac{N}{D} .
$$

We thus have for $y=N / D$ the situation $D, N>0$ at and around the steady state. This assumption implies - though goods demand is clearly swage-led، (which - up to its extent is natural in the case where the marginal propensity to consume is larger than the investment parameter $\beta_{r}$ ) - that the IS-curve depends negatively on the wage share $v .{ }^{\mathrm{II}}$ In this case we therefore get that effective (not aggregate) demand, given by the ratio $y$, depends negatively on the wage share $v$ (as one would not expect it to be true in an economy where goods demand is wage led).

Note that we are considering in this section the case where the responsiveness of investment to a subnormal profit rate, as measured by the parameter $\beta_{r}$, is less than one. The investment behavior of firms is thus still of a fairly tranquil type. ${ }^{\text {I2 }}$

The considered situation moreover implies a positive dependence of $y$ on the debt to capital ratio $\lambda_{w}^{a}$ (around the steady state of the model, to be discussed below). For the partial derivatives of the functions $y, l=v y, r$ with respect to $v$ we obtain in detail:

$$
\frac{\partial y}{\partial v}=\frac{N D_{v}}{D^{2}}=-y \frac{c-\beta_{r}}{D}<0, \frac{\partial v y}{\partial v}=y \frac{\beta_{r}-1}{D}<0, \frac{\partial r}{\partial v}=-y \frac{c-1}{D}<0 .
$$

For the derivative $\frac{\partial y}{\partial \lambda_{w}^{a}}$ we in addition always have

$$
\frac{\partial y}{\partial \lambda_{w}^{a}}=\frac{c i}{D}>0, \quad \frac{\partial r}{\partial \lambda_{w}^{a}}=\frac{c i}{D}(1-v)>0,
$$

which transforms itself directly into corresponding derivatives for $v y, r=(1-v) y-\delta$. The above partial derivatives with respect to the state variable $v$ show in particular that these derivatives do not depend on the interest rate $i$ at the steady state, so that the influence of $i$ on the Jacobian of the dynamics is only given when the term $\frac{\partial y}{\partial \lambda_{w}^{a}}=c i$ / $D$ is involved. Note that we will use for brevity $y_{v}$ in place of $\frac{\partial y}{\partial v}$, etc. in the following.

The negative sign of the derivative $y_{v}$ shows the somewhat striking result that goods market dynamics are profit-led, though aggregate demand per unit of capital is clearly wage-

II This situation always holds if the marginal propensity to spend is larger than one and aggregate demand is wage-led, since an increase in the wage share must then be compensated by a decrease in output in order to restore goods market equilibrium.

I2 See Section 5 for the consideration of the case $c<\beta_{r}$. 
led: $y_{v}^{d}=\left(c-\beta_{r}\right) y>0$. This indicates that empirical findings on the role of income distribution on the market for goods (where we in fact can only observe empirically the interactions of demand with supply) which state that the goods market behavior is profit led (decreases with increases in the wage share) can be completely in line with the unobservable fact that planned goods demand depends positively on the wage share (is wage led). We note also that in the extreme case here considered we have a positive effect of increases in the loan rate on the state of the goods market. This rather counterintuitive result is a direct consequence of the very excessive consumption case considered here.

The situation we have considered so far gives rise to the following stability proposition.

Proposition I: Stability for a `normak range of parameter values

Assume that $i$ is sufficiently small and $\beta_{\text {we }}$ sufficiently large. Then: The steady state of the dynamics (I6) - (I8) is locally attracting for all empirically relevant parameter sizes of the model.

This result is maybe not so surprising as it may first be thought to apply to a significantly debt driven economy. Default so far only works as if it were a 'gift ( debt relief) from the side of asset holders, given to worker households, since the assumed bankruptcy of part of these latter households (the loss of their homes ${ }^{13}$ ) does not yet feedback into the considered dynamics (since the law of motion for $k_{w}$ is only an appended one). Moreover, the Goodwin part of the model (the interaction of the state variables $v$ and $l$ is here of a convergent type, while the only destabilizing feedback loop is the one between the state variables $v$ and $\lambda_{w}^{a}$ through their positive interaction. ${ }^{14}$ Debt has a positive effect on the growth rate of the wage share and the latter may have a positive effect on the time rate of change of the debt to capital ratio. But this accelerating mechanism is not yet dominant in the overall interaction of the three state variables that are here considered.

There is however an element in the considered situation that can lead the investigated dynamics into a situation where the steady state becomes unstable and where the assumed degree of wage flexibility will lead the economy towards explosive fluctuations. This case comes about when it is assumed that the considered excessive overconsumption stimulates asset holders to invest into real capital at a rate $\beta_{r}$ that is larger than $c$. In this case we get $y_{v}>0$ and thus a wage-led goods market behavior. Since the stability proof of this section demanded for a degree of wage flexibility $\beta_{w e}$ that is sufficiently high, it is then very likely that the trace of $J$ :

$$
\beta_{w e} y_{v} v_{o}-(c-1) i-\beta_{r} r_{\lambda_{w}^{a}} \lambda_{w}^{a}-\left(n+\varphi_{d}\right)
$$

becomes positive and the steady state becomes a repelling one.

I3 And their living in cars or tents as it now happens in the US economy.

I4 As shown through the entries $J_{13}, J_{31}$, see the mathematical appendix. 


\subsection{The unstable mildly excessive overconsumption case}

We consider now the only mildly excessive wage-led case

$$
\beta_{r}<1<c<1+\left(1-\beta_{r}\right)\left(1-v_{o}\right) / v_{o} .
$$

In this case we now have a significantly different situation where $D, N<0$ in the goods market equilibrium expression $y=N / D$ at the steady state. For the partial derivatives of the functions $y, v y, r$ with respect to $v$ we therefore now get: ${ }^{\text {I5 }}$

$$
y_{v}=-y \frac{c-\beta_{r}}{D}>0,(v y)_{v}=y \frac{\beta_{r}-1}{D}>0, r_{v}=-y \frac{c-1}{D}>0 .
$$

And for the derivative we in addition always have

$$
(v y)_{\lambda_{w}^{a}}=\frac{c i}{D}<0
$$

which transforms itself directly into corresponding derivatives for $v y, r=(1-v) y-\delta$. Note that all partial derivatives have now the opposite sign as compared to the overconsumption case considered previously. The sign of the derivative $y_{v}>0$ now shows the normal result that goods market reaction appears as wage-led when aggregate goods demand per unit of capital is wage-led: $y_{v}^{d}=\left(c-\beta_{r}\right) y>0$. Moreover, interest rate effects, i.e., rising loan rates are now contractive: $y_{i}>0$ (instead of activity increasing) as one would expect it intuitively in a debt driven economy. This gives rise to the Proposition 2.

Proposition 2: Instability of the mildly excessive consumption case

Assume $\beta_{r}<1<c<1+\left(1-\beta_{r}\right)\left(1-v_{o}\right) / v_{o}$. The steady state of the dynamics (I6)-(I8)

is locally repelling for empirically plausible choices of the parameter values of the model.

The case of a mildly excessive overconsumption in the sector of workers' households does therefore not represent a viable situation, in particular since the previously stable dynamics of the Goodwin substructure with its state variables $v, l$ have now become a totally unstable one. This is further exemplified by the following proposition which states that even very low loan rates $i$ cannot be of help in the considered case.

Proposition 3: Instability for small values of the loan rate

Assume $i=0<n+\varphi_{d}$. Then: The steady state of the dynamics (I6) - (I8) is locally repelling. This then also holds for all loan rates chosen sufficiently small.

So far we have investigated the parameter situations A and B shown in Figure I. We have found out that case $B$ (the excessive overconsumption case) represents by and large a stable performance of the economy, while case A (the mildly excessive overconsumption case) does not provide a viable situation from the economic point of view. The overall conclusion here therefore is that investment should be stimulated at least to the extent such that $\beta_{r}>\frac{1-c v_{o}}{1-v_{o}}$ holds true, for example through a policy that lowers the loan rate to a suf-

I5 Note that $Y_{Y}^{d}<1$ holds now. 
ficient degree such that this inequality can be ensured (assuming that the parameter depends negatively on the loan rate i).

Figure I: A summary of the stability scenarios for a varying parameter

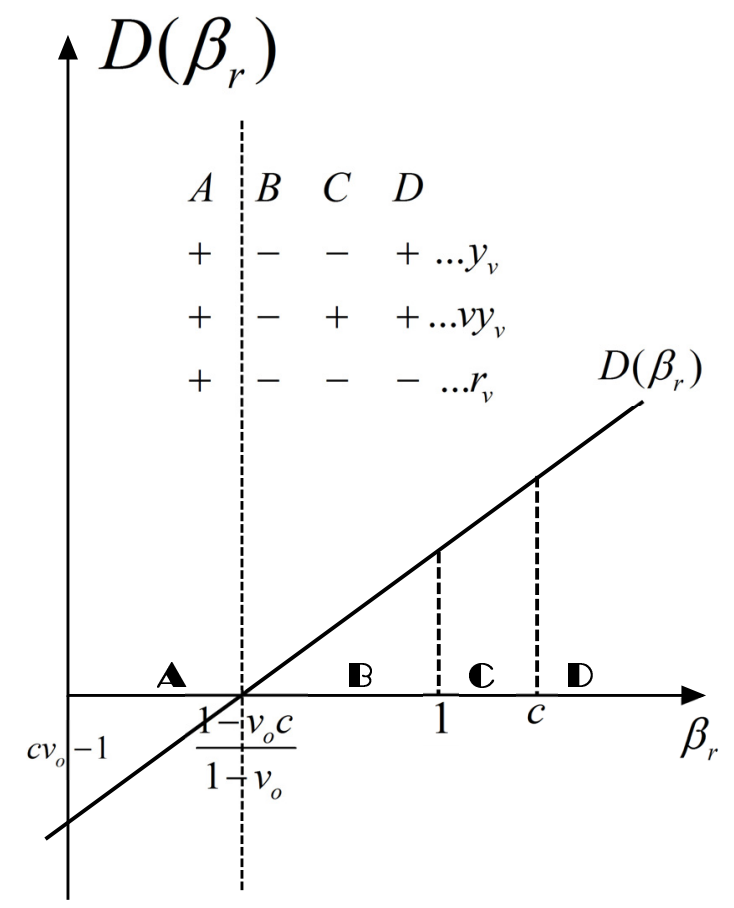

Yet this may stimulate investment decisions to such an extent that situation $\mathrm{C}$ or even situation $\mathrm{D}$ becomes established. In situation $\mathrm{C}$ the entry $J_{31}$ in the matrix $J$ becomes unambiguously positive which increases the negative forces in the Routh-Hurwitz coefficient $a_{2}$. The overall stability situation however is not clear in this case, while it is fairly obvious that case $\mathrm{D}$ represents an unstable case, in particular since we now have a positive $J_{11}$ that will destabilize the economy if wages are or are becoming sufficiently flexible with respect to demand pressure on the market for labor.

We conclude that strong consumption as well as investment behavior tends to destabilize the situation we analyzed in Section 3.

In this case $\beta_{r}>c$ and we thus again get $y_{v}>0$ and thus a wage-led goods market behavior. Since the stability proof of Section 3 demanded for a degree of wage flexibility $\beta_{w e}$ that is sufficiently high it is then very likely that the trace of $J$ :

$$
\beta_{w e} y_{v} v_{o}-(c-1) i-\beta_{r} r_{\lambda_{w}^{a}} \lambda_{w}^{a}-\left(n+\varphi_{d}\right)
$$

then becomes positive and the steady state becomes a repelling one. We will investigate such a situation in the next section. 


\section{Credit rationing, reduced consumption and the emergence of mortgage crises}

Beginning from the case of excessive overconsumption just discussed, we assume now that asset holders stop lending to workers due to an exogenous change in their risk perception concerning workers' debt. One may for example assume here that the loan rate $i$ (and with it on the macro-level inevitably - though unintentionally - the default rate $\varphi_{d}$ ) has been increasing in a stepwise fashion until a level of the default rate has been reached that persuades asset holders to stop their lending to worker households. The immediate consequence is that the propensity to consume is forced to the value 1 (or close to one if some lending still goes on, the case we considered in the preceding section). In the following we therefore consider the stability features of case $c \leq 1$.

We start the investigation from the case $c \leq 1$, but sufficiently close to 1 . We have in this situation $D<0$, but now in addition also $\lambda_{w o}^{a}<0$. The assumed shock to workers' propensity to consume has an immediate effect on goods market equilibrium

$$
y=\frac{c i \lambda_{w}^{a}+\beta_{r}\left(\delta+r_{o}\right)-n-\delta}{\left(c-\beta_{r}\right) v+\beta_{r}-1}, \quad\left(c-\beta_{r}\right) v+\beta_{r}-1<0
$$

now.

The change in IS-equilibrium resulting from a change in the parameter $c$ is obtained by taking the total differential of the IS equation as follows:

$$
d c \cdot v y+\left[\left(c-\beta_{r}\right) v+\beta_{r}-1\right] \cdot d y=d c \cdot i \lambda_{w}^{a}=0 .
$$

Rearranging this equation appropriately then gives

$$
\frac{d y}{d c}=\frac{-v y+i \lambda_{w}^{a}}{\left(c-\beta_{r}\right) v+\beta_{r}-1} .
$$

Viewed from the situation $c=1$ we thus get that a positive shock to $c$ increases economic activity (since the steady state value of $\lambda_{w}^{a}$ is zero at this position), i.e., in reversed terms, the jump to a marginal propensity to consume of one in the above considered crisis situation is contractive and would lead the economy into the deflationary region (as far as the real wage dynamic is concerned) if it has been at its steady state position initially.

As steady state position we now have

$$
y_{o}=y^{p} l_{o}=y_{o} / \bar{e}, y_{w o}=y_{o}-\delta-n, \lambda_{w o}^{a}=0, v_{o}=y_{w o}
$$

and for the employed partial derivatives we get in this case (since the denominator in the IS curve is now negative):

$$
y_{v}=-y \frac{1-\beta_{r}}{D}>0, \quad(v y)_{v}=y \frac{\beta_{r}-1}{D}>0, \quad r_{v}=0 .
$$

For the derivative $(v y)_{\lambda_{w}^{a}}$ we in addition now have a negative sign: $(v y)_{\lambda_{w}^{a}}=\frac{c i}{D}<0$.

Proposition 4: Full credit rationing implies a repelling steady state 
Assume $c=1$. Then: The steady state of the dynamics (I6) - (I8) is surrounded by explosive

forces. This also holds for all marginal propensities $c$ chosen sufficiently close to 1 .

We get from the proof of Proposition 4 (see appendix) for the entries of the Jacobian matrix $J$ of the dynamics at the steady state as sufficient condition for instability the inequality:

$$
a_{2}<0 \text { if and only if }-J_{12} J_{21}<-J_{11} J_{33}+J_{13} J_{31} \text {. }
$$

This condition states that the stability created by the profit-led Goodwin (1967) subcycle of the model (the interaction between the wage share and the employment rate, here expressed through the labor intensity ratio $l$ ) is overcome by the destabilizing feedback chain between the wage share $v$ and the debt to capital ratio $\lambda_{w}^{a}$. Of course, further instability scenarios may come about through the trace of the matrix $J$ if wages are sufficiently flexible with respect to the labor market utilization gap, and through the determinant of $J$ (if $J_{23} J_{31}$ becomes dominant). The case where workers save in order to reduce their prevailing level of debt does therefore not at all represent a situation where recovery and convergence towards the steady state of the economy can be expected.

\section{Monetary policy in a mortgage crisis}

In order to determine what monetary policy could do in the situation we discussed in the preceding section we now consider the formulated rise in the loan rate i that triggered the crisis in somewhat more detail. We consider for this purpose the situation shown in Figure 2.

The basic assumption in Figure 2 is that the default rate $\varphi_{d}$ is an increasing function of the loan rate $i$ and this in the strictly convex form shown in Figure 2. We add to this function the term $r_{o}=n$ and obtain an expression (as a function of the loan rate $i$ ) that shows the minimum loan rate needed to obtain as rate of return on loans the (steady state) rate of profit of firms. We next assume that - under normal conditions - the actual loan rate is decreased if it exceeds the given rate of profit plus the default rate (viewed as rate of depreciation of loans). It therefore tends towards the minimum loan rate $i_{o}$ when one starts from the initial situation $A$ for example. Such an adjustment thus leads in the limit to the loan rate $i_{o}$ and thus to a tranquil situation where the loan rate as well as the default rate are small, a situation that is attracting smaller as well as larger loan rates in an asymptotically stable way. Yet as the Figure 2 shows the basin of attraction of the loan rate $i_{o}$ is limited to the right by the loan rate $i_{1}$, i.e., the considered stable adjustment process becomes an unstable one to the right of this rate. 
Figure 2: Loan rate adjustment dynamics

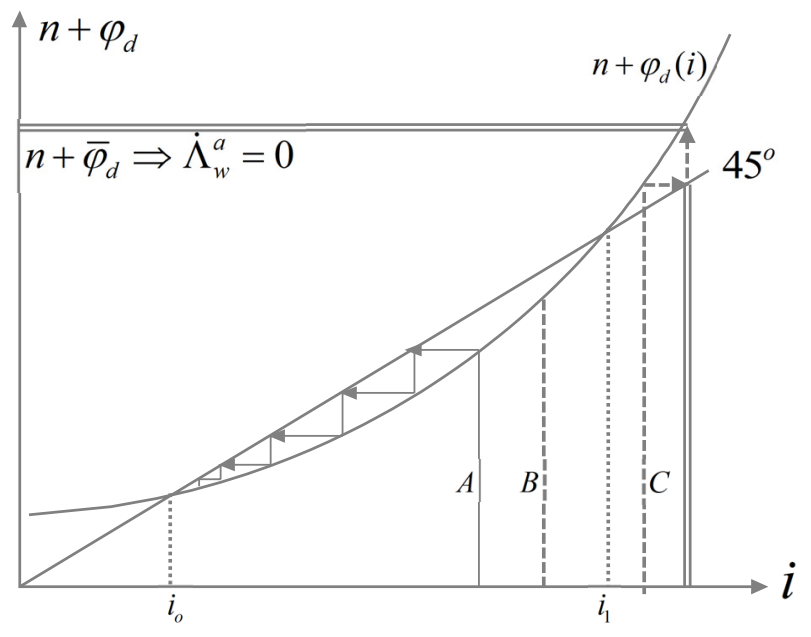

Consider now the case $B$ in Figure 2. We here assume that external events have led to this increase in the loan rate (maybe in a stepwise fashion) since asset holders now insist - in place of the above adjustment process - on a loan rate that guarantees a higher rate of return on their loans than the one in the sector of firms. Assuming that this downward rigidity of the loan rate eventually leads (through exogenously driven further actions of asset holders) to a loan rate that is higher than $i_{1}$ and which pushes the financial market of the economy into the unstable region where the loan rate gives rise to an endogenous upward spiraling process $C$ (since the rate of return on loans is now lower than the rate of profit and thus now inducing asset holders to further increases in $i$ ). We assume however that this process comes to an end at the value determined by the double line, since asset holders then stop giving credit to workers as discussed in the preceding section (due to the high default rate that accompanies the then existing loan rate). The economy is then trapped into a situation where the loan rate as well as the default rate are very high and where the propensity to buy houses is zero and the overall propensity to consume therefore less than one.

In this still very basic scenario (as far as mortgage crises are concerned) the policy to be adopted by the central bank (which is now assumed to enter the stage) is still a simple one. The first step in the solution to the credit crisis simply is to buy an amount of bad loans that allows for a loan rate $i$ smaller than $i_{1}$ for the bad loans $\bar{\varphi}_{d}(i)-\varphi_{c}$ that then remain in the sector of asset holders. This increases the reserves of asset holders and must be conditioned on their acceptance of the minimum loan rate (on the strictly convex curve in Figure 2) that is consistent with the debt default situation they are then facing. If this acceptance is followed in all subsequent steps we may then expect that the process leading back to the loan rate $i_{o}$ becomes again into being, now in the situation where the overall propensity of workers to consume is less than one (and where debt of workers is reduced in each point in time through their now positive savings rate). The question then becomes how the pri- 
vate sector of the economy (the real part of the economy and the process of debt de-cumulation) behaves in the situation obtained in this way.

The downward correction of the loan rate just considered is however not yet sufficient in order to stabilize the economy, since the Jacobian we considered at the end of the preceding section is plagued by a wage-led situation as far as the entry $J_{11}$ is concerned and exhibits a destabilizing feedback mechanism through the entries $J_{13}, J_{31}$ which are both negative and thus endangering the positivity of $a_{2}$. In order to get a situation where the stability of the private sector can be ensured we consider again the sign of the determinant of $J$ as given by the Expression (2I). We continue to investigate the case $c<1, \beta_{r}<1$, i.e., the situation where $D<0$ holds true. We assume now a situation where $1>\beta_{r}>c>0$ has been established, e.g. through forced savings of worker household, based on their debt the central bank is now holding.

Simple empirical estimates as they were considered with Equation (2I) - or just the assumption that $\beta_{r}$ is sufficiently close to 1 - then ensure that the determinant of the matrix $J$ is negative. Moreover we have in this case $y_{v}<0,(v y)_{v}>0, r_{v}<0, y_{\lambda_{w}^{a}}<0, r_{\lambda_{w}^{a}}<0$ and thus get in this case as sign distribution in the Jacobian for the dynamics at the steady state:

$$
J=\left(\begin{array}{ccc}
k y_{v} v_{o} & -k \bar{e} v_{o} & k y_{\lambda_{w}^{a}} v_{o} \\
-\beta_{r} r_{v} l_{o} & 0 & -\beta_{r} r_{\lambda_{w}^{a}} l_{o} \\
(c-1)(v y)_{v}-\beta_{r} r_{v} \lambda_{w o}^{a} & 0 & (c-2) i-\beta_{r} r_{\lambda_{w}^{a}} \lambda_{w o}^{a}
\end{array}\right)=\left(\begin{array}{ccc}
- & - & - \\
+ & 0 & + \\
- & 0 & -
\end{array}\right) .
$$

This immediately implies the stability of the Goodwin part of the model (the interaction of $v, l)$.

\section{Proposition 5: Stability for small values of $i$ and flexible wage adjustment}

$$
\begin{aligned}
& \text { Assume that the parameter } k \text { is sufficiently large and; } i=n+\varphi_{d}(i)>0 \text { sufficiently small. } \\
& \text { Then: The steady state of the dynamics (IG) - (I8) is locally attracting. }
\end{aligned}
$$

In view of what we have shown with respect to Figure 2 the first objective of the central bank thus should be to support keeping the loan rate sufficiently low. In a next step consumption should be discouraged or/and investment encouraged. One may for example assume that the value of $\beta_{r}$ depends negatively on the loan rate $i$. Improving the investment climate to a sufficient degree may therefore be one policy option of the central bank.

The central bank starts by buying a fraction $\alpha$ of the default volume, the bad loans $\varphi_{d} \lambda_{w}^{a}$. This increases the disposable income of asset holders ${ }^{16}$ and leads to an increase of either their reserve holding $R$ (which we have ignored so far) or their investment, leading to an

I6 Note that we here assume for the disposable income of asset holders the equation: $Y_{c}=r K+i \Lambda_{w}^{a}+\alpha \varphi_{d}(i) \Lambda_{w}^{a}$.

This however does not alter the steady state of the model, since aggregate defaults remain the same (implying no change in the law of motion for $\lambda_{w}^{a}$ ), though part of them are now held by the central bank. 
adjustment of the parameter $\beta_{r}$ that may become permanent even if the central bank stops buying bad loans. In the case it continues to do so we have the situation that bad loans no longer lead to bankruptcy (loss of their homes) of the corresponding worker households. One may assume in addition that the central bank starts demanding some low interest payment from them, but we do not consider this here explicitly.

Since asset holders now face less bad loans $(1-\alpha) \varphi_{d} \lambda_{w}^{a}-$ due to the bail-out exercised by the central bank - we have moreover that the loan rate falls, as it was intended by the central bank. This induces a further reduction in the amount of bad loans and thus improves the credit situation worker households are facing. There is less debt default and less bankruptcy (which was accompanied by the destruction of workers' homes) and cheaper credit for worker households and - as we have argued - increased robustness in the stability scenario of the private sector that this policy implies.

We summarize the stability analysis of this paper in Figure 3 which provides just another representation of what we have already considered in Figure I. In Figure 3 we show this from the perspective of the slope of the IS-curve with respect to the wage share $v$ which clearly shows where the economy may be a viable one and where not. Note that the viability domain is exactly the one where our Keynes-Goodwin model seems to be profit led in the way comparable to the Goodwin (1967) growth cycle model.

Figure 3: An alternative summary of the stability scenarios for a varying parameter

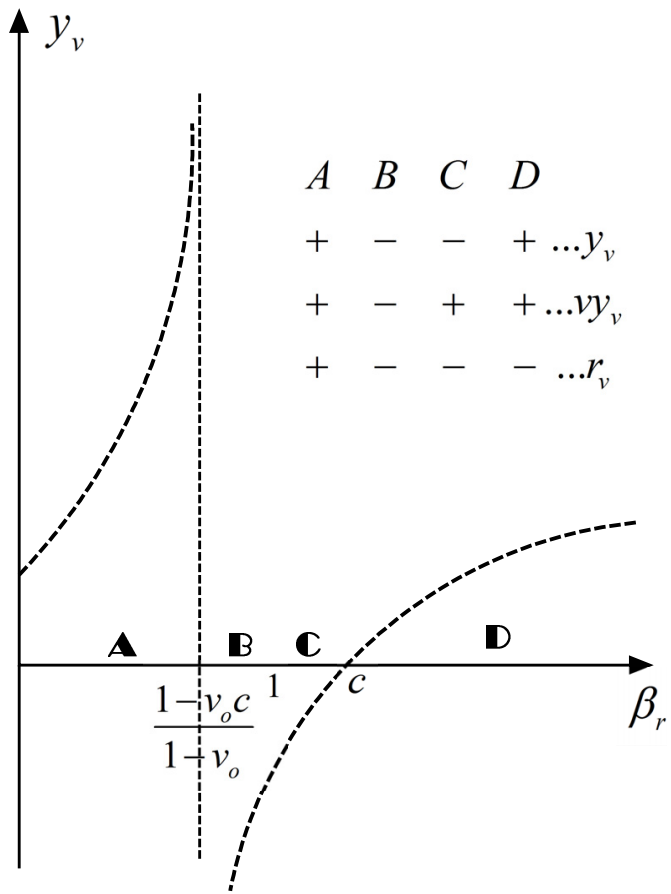


The general form of the signs of the matrix in the domains $\mathrm{B}$ and $\mathrm{C}$ is

$$
J=\left(\begin{array}{ccc}
- & - & + \\
- & 0 & - \\
? & 0 & -
\end{array}\right) .
$$

The domains $\mathrm{B}$ and $\mathrm{C}$ are therefore the ones where the economy may be able to reproduce itself in the long-run. The care of the central bank should then only be to keep the investment climate in an appropriate range and the loan rate on workers debt reasonably low. Nevertheless there are still a variety of cases conceivable where there is a latent tendency towards instability (approached through investment weaknesses or investment booms), since in particular the regime of overconsumption may not be the best of all accumulation regimes.

\section{Conclusions}

In this paper we considered an initial situation of stable excessive overconsumption of worker households, and from there a situation of strict credit rationing by asset holders. We then considered actions that rescue the economy from this last instability scenario and that imply a solution that leads the economy back to economic stability based on investment stimuli and monetary policy regarding the loan and the default rate on the credit market.

In this latter type of economy, the loan rate will fall to a low value again and workers' debt will reduce in time through their now positive savings. In the limit, this economy would even converge to a situation where workers are lending to asset holders, since they have a positive savings rate in the steady state. Yet, we view this phase as representing only a transient phase where the economy recovers to a certain degree from too high mortgage debt to its normal functioning, maybe subsequently followed again by the emergence of some behavior which leads the macroeconomy back to the stable overconsumption situation we started from. The sequence of events analyzed in this paper may repeat itself in actual historical events.

In view of the quotation from Minsky's work at the beginning of this paper it may therefore well be - if this transient phase is long enough - that the first type of excessive overconsumption reappears and that the whole process starts again, though possibly in a different historical garment. 


\section{Mathematical appendix}

\section{Proof of Proposition I:}

The Jacobian of the dynamics (I6) - (I8) for the considered special case reads: ${ }^{17}$

$$
J=\left(\begin{array}{ccc}
k y_{v} v_{o} & -k \bar{e} v_{o} & k y_{\lambda_{w}} v_{o} \\
-\beta_{r} r_{v} l_{o} & 0 & -\beta_{r} r_{\lambda_{w}^{a}} l_{o} \\
(c-1)(v y)_{v}-\beta_{r} r_{v} \lambda_{w o}^{a} & 0 & (c-2) i-\beta_{r} r_{\lambda_{w}^{a}} \lambda_{w o}^{a}
\end{array}\right) .
$$

For the sign of the determinant of this Jacobian we therefore have to investigate the sign of the following determinant

$$
|J|=\frac{y i}{D}\left|\begin{array}{ccc}
-\left(c-\beta_{r}\right) & -\bar{e} & \frac{c}{D} \\
c-1 & 0 & -\left(1-v_{o}\right) \frac{c}{D} \\
(c-1)\left(\beta_{r}-1\right)+\beta_{r}(c-1) \lambda_{w o}^{a} & 0 & (c-2)-\beta_{r}\left(1-v_{o}\right) \frac{c}{D} \lambda_{w o}^{a}
\end{array}\right| .
$$

The sign of this determinant is therefore equal to the sign of its following sub-determinant:

$$
\left|\begin{array}{cc}
1 & -\left(1-v_{o}\right) \frac{c}{D} \\
\beta_{r}-1+\beta_{r} \lambda_{w o}^{a} & (c-2)-\beta_{r}\left(1-v_{o}\right) \frac{c}{D} \lambda_{w o}^{a}
\end{array}\right| .
$$

For the sign of the latter determinant we get the expression

$$
c-2-\beta_{r}\left(1-v_{o}\right) \frac{c}{D} \lambda_{w o}^{a}+\left(1-v_{o}\right) \frac{c}{D}\left(\beta_{r}-1+\beta_{r} \lambda_{w o}^{a}\right)
$$

which finally simplifies to

$$
c-2+\left(1-v_{o}\right) \frac{c}{D}\left(\beta_{r}-1\right) .
$$

This expression is negative if there holds $\beta_{r} \leq 1$ and if we assume that $c<4$ / 3 which surely is a plausible upper limit for the marginal propensity to consume. ${ }^{18}$

I7 $i=n+\varphi_{d}$. Note that the $c-2$ in the Jacobian $J$ is due to this assumption. Note also that we use the abbreviation $k=\beta_{w e} / l_{o}$.

I8 In the case $c>\beta_{r}>1$ we get from $D=\left(c-\beta_{r}\right) v_{o}+\beta_{r}-1>\beta_{r}-1>0$ the inequality $c-2+\left(1-v_{o}\right) \frac{c}{D}\left(\beta_{r}-1\right)<c-2+\left(1-v_{o}\right) c<1.5 c-2<0$ if $c<4 / 3, v_{o}>0.5$ holds true, i.e., we get a negative determinant $|J|\left(=-a_{3}\right)$ for all empirically plausible parameter values $c>1$ and 
The assumption on the size of $c$ also implies that the trace of $J\left(=-a_{1}\right)$ is negative as well. For the sum of the principal minors of order two

$$
a_{2}=\frac{k y_{o} v_{o}(1-c)}{D} \beta_{r} y_{o}+\left|\begin{array}{ll}
J_{11} & J_{13} \\
J_{31} & J_{33}
\end{array}\right|
$$

we get from the above with respect to the term on the right hand side:

$$
J(2)=\left|\begin{array}{ll}
J_{11} & J_{13} \\
J_{31} & J_{33}
\end{array}\right|=\frac{k y_{o} v_{o}(1-c)}{D} \frac{i}{D}\left|\begin{array}{cc}
-\left(c-\beta_{r}\right) /(c-1) & c \\
\beta_{r}\left(1+\lambda_{w o}^{a}\right)-1 & D(c-2)-\beta_{r} c\left(1-v_{o}\right) \lambda_{w o}^{a}
\end{array}\right| .
$$

A sufficient assumption for obtaining $a_{2}>0$ (much more than is actually needed) therefore is $\beta_{r}\left(1+\lambda_{w o}^{a}\right)<1$. It seems however more appropriate to assume that the loan rate $i$ is chosen sufficiently small, ${ }^{19}$ such that - should the considered determinant be negative - it is dominated by the first principal minor $\frac{k y_{o} v_{o}(1-c)}{D} \beta_{r} y_{o}$.

The remaining Routh-Hurwitz condition for local asymptotic stability is:

$$
a_{1} a_{2}-a_{3}>0 \text { where } a_{1}=-\operatorname{trace} J, a_{3}=-|J| .
$$

The determinant of $J$ is however dominated by the remaining terms in $a_{1} a_{2}$, if the adjustment speed of money wages $\beta_{w e}$ is chosen sufficiently large, since it enters $a_{1}, a_{2}, a_{3}$ linearly with a positive slope and thus in $a_{1} a_{2}$ through a positively sloped quadratic term.

We therefore in sum have

$$
a_{1}>0, a_{2}>0, a_{3}>0, a_{1} a_{2}-a_{3}>0
$$

for the coefficients of the characteristic polynomial of the matrix $J$ (the Routh-Hurwitz conditions) and have thus shown the local asymptotic stability of the steady state of the model. ${ }^{20}$ This concludes the proof of Proposition I.

\section{Proof of Proposition 2:}

In the case of Proposition 2 we have for the determinant of the Jacobian:

$$
|J|=\left|\begin{array}{ccc}
0 & -k \frac{y_{o}}{l_{o}} v_{o} & 0 \\
-\beta_{r} r_{v} l_{o} & 0 & -\beta_{r} r_{\lambda_{w}^{a}} l_{o} \\
(c-1)(v y)_{v} & 0 & (c-1) i-\left(n+\varphi_{d}\right)
\end{array}\right| \approx\left|\begin{array}{ccc}
0 & - & 0 \\
- & 0 & + \\
- & 0 & -n-\varphi_{d}
\end{array}\right|,
$$

$v_{o}=\frac{y_{o}-\delta-n}{y_{o}}$.

I9 See $J_{31}$ in the last formula.

20 This also holds true for all $1 \geq \beta_{r}<c$. 
and can thus assume that this determinant is always positive for values of $c-1, i$ that are of empirical relevance (under a relatively normal working of the economy). This assumption does also no longer imply that the trace of $J$ is negative. And for the sum of the principal minors of order two we get from the above:

$$
\begin{aligned}
& a_{2}=\left|\begin{array}{cc}
k y_{v} v_{o} & -k \frac{y_{o}}{l_{o}} v_{o} \\
-\beta_{r} r_{v} l_{o} & 0
\end{array}\right|+\left|\begin{array}{cc}
k y_{v} v_{o} & k y_{\lambda_{v}^{a}} v_{o} \\
(c-1)(v y)_{v}-\beta_{r} r_{v} \lambda_{w}^{a} & (c-1) i-\beta_{r} r_{\lambda_{w}^{a}} \lambda_{w}^{a}-\left(n+\varphi_{d}\right)
\end{array}\right|, \\
& \text { i.e., } \\
& \approx\left|\begin{array}{cc}
- & -k \frac{y_{o}}{l_{o}} v_{o} \\
\beta_{r} \frac{y_{o}(c-1)}{D} l_{o} & 0
\end{array}\right|+\frac{k y_{o} v_{o} c i}{D^{2}}\left|\begin{array}{cc}
-\left(c-\beta_{r}\right) & 1 \\
(c-1)\left[\beta_{r}\left(1+\lambda_{w o}^{a}\right)-1\right] & -\beta_{r}\left(1-v_{o}\right) \lambda_{w o}^{a}-\frac{n+\varphi_{d}}{c i D}
\end{array}\right| .
\end{aligned}
$$

We conjecture here that also the second determinant of this expression is very likely to be negative. The validity of the conditions

$$
a_{1}>0, a_{2}>0, a_{3}>0, a_{1} a_{2}-a_{3}>0
$$

on the coefficients of the characteristic polynomial of the matrix $J$ (the Routh-Hurwitz conditions) is therefore in general hurt from various perspectives, which implies the proposition.

\section{Proof of Proposition 3:}

It is easily shown that the sign structure of the Jacobian is in the case $i=0$ of the qualitative form:

$$
J=\left(\begin{array}{ccc}
k y_{v} v_{o} & -k \bar{e} v_{o} & k y_{\lambda_{w}^{a}} v_{o} \\
-\beta_{r} r_{v} l_{o} & 0 & -\beta_{r} r_{\lambda_{v}^{a}} l_{o} \\
(c-1)(v y)_{v}-\beta_{r} r_{v} \lambda_{w o}^{a} & 0 & (c-2) i-\beta_{r} r_{\lambda_{w}^{a}} \lambda_{w o}^{a}
\end{array}\right)=\left(\begin{array}{ccc}
+ & - & 0 \\
- & 0 & 0 \\
? & 0 & -
\end{array}\right) .
$$

This immediately implies the instability of the (wage-led!) Goodwin part of the model (the interaction of $v, l$ ), since the subsystem corresponding to the state variables $v, l$ does not depend on the third state variable $\lambda_{w}^{a}$. The real parts of the eigenvalues are therefore not all negative, a fact that extends to all $i$ sufficiently small, since the eigenvalues depend continuously on the parameters of the model.

\section{Proof of Proposition 4:}

The Jacobian of the dynamics at the steady state now reads as follows: 


$$
J=\left(\begin{array}{ccc}
k y_{v} v_{o} & -k \bar{e} v_{o} & k y_{\lambda_{w}} v_{o} \\
-\beta_{r} r_{v} l_{o} & 0 & -\beta_{r} r_{\lambda_{w}} l_{o} \\
(c-1)(v y)_{v}-\beta_{r} r_{v} \lambda_{w o}^{a} & 0 & (c-2) i-\beta_{r} r_{\lambda_{w}} a_{w o}^{a}
\end{array}\right)=\left(\begin{array}{ccc}
k y_{v} v_{o} & -k \frac{y_{o}}{l_{o}} v_{o} & k y_{\lambda_{w}^{a}} v_{o} \\
0 & 0 & -\beta_{r} r_{\lambda_{w}^{a}} l_{o} \\
0 & 0 & -\left(n+\varphi_{d}\right)
\end{array}\right) .
$$

This gives in signs the qualitative structure:

$$
J=\left(\begin{array}{ccc}
+ & - & - \\
0 & 0 & + \\
0 & 0 & -
\end{array}\right) .
$$

This immediately implies the instability of the dynamics, since two of the principal minors of order 2 are zero while the third one is negative, i.e., there holds $a_{2}>0$. This proves the proposition.

Note that the determinant of the Jacobian $J$ is zero in the situation $c=1$, and nonzero (positive or negative) for $c<1$. There will be one eigenvector direction where the dynamics is on a very slow track, and there is in addition always one unstable (and one stable ${ }^{21}$ root, where the former drives the system away from the steady state.

In the case of an arbitrary $c \in\left(\beta_{r}, 1\right)$ we get for the Jacobian the qualitative structure:

$$
J=\left(\begin{array}{ccc}
+ & - & - \\
+ & 0 & + \\
- & 0 & -
\end{array}\right),
$$

since there holds

$$
J_{31}=(c-1) y \frac{\beta_{r}-1}{D}-\beta_{r}(-y) \frac{c-1}{D} \lambda_{w o}^{a}=(c-1) y \frac{1-\beta_{r}}{|D|}+\beta_{r} y \frac{1-c}{|D|} \lambda_{w o}^{a}<0
$$

due to $\lambda_{w o}^{a}<0$.

\section{Proof of Proposition 5:}

Proof: We have already $a_{1}=-\operatorname{trace} J>0, a_{3}>0$. Choosing $k$ sufficiently large also gives $a_{2}>0$ if the entries in the last column of $J$ are made sufficiently small by choosing a low value of the loan rate $i$. The latter situation also ensures $a_{1} a_{2}-a_{3}>0$, since $a_{3}$ can be made small relative to $a_{1} a_{2}$ in this way. 


\section{References}

Ashcraft, A., Schuermann, T. (2007): Understanding the securitization of Subprime Mortgage Credit, Federal Reserve Bank of New York Staff Report 318.

Dell'Ariccia, G., Igan, L., Laeven, A. (2008): Credit booms and lending standards: Evidence from the Subprime Mortgage Market, IMF Working Paper, Nr. 08/ı66.

Dutt, A.K. (2003): Conspicuous consumption, consumer debt and economic growth, in: Setterfield, M. (ed.), Interactions in Analytical Political Economy, Armonk, New Jersey: M.E. Sharpe, $155-78$.

Dutt, A.K. (2006): Maturity, stagnation and consumer debt: A Steindlian approach, in: Metroeconomica, $57(3), 339-364$.

Flaschel, P., Krolzig, H.-M. (2006): Wage-price Phillips curves and macroeconomic stability: Basic structural form, estimation and analysis, in: Chiarella, C., Flaschel, P., Franke, R., Semmler, W. (eds.), Quantitative and Empirical Analysis of Nonlinear Dynamic Macromodels, Contributions to Economic Analysis, Amsterdam: Elsevier, 7 - 47.

Flaschel, P. (2009): The Macrodynamics of Capitalism, Elements of a Synthesis of Marx, Keynes and Schumpeter, Berlin: Springer.

Goodwin, R.M. (1967): A growth cycle, in: Feinstein, C.H. (ed.), Socialism Capitalism and Growth, Cambridge: Cambridge University Press, $54-58$.

Minsky, H. (1982): Can ,It happen again?, New York: ME Sharpe.

Minsky, H. (1992): The Capital Development of the Economy and the Structure of Financial Institutions, Bard College: The Levy Economics Institute.

Palley, T. (1994): Debt, aggregate demand and the business cycle. An analysis in the spirit of Kaldor and Minsky, in: Journal of Post Keynesian Economics, I6(3), 37I - 90.

Taylor, L., O’Connell, S. (1989): A Minsky crisis, in: Semmler, W. (ed.), Financial Dynamics and Business Cycles, New York: M.E. Sharpe, 3 - I7. 